\title{
Research
}

\section{Clinical features of bladder cancer in primary care}

\begin{abstract}
Background

Bladder cancer accounts for over 150000 deaths worldwide. No screening is available, so diagnosis depends on investigations of symptoms. Of these, only visible haematuria has been studied in primary care.
\end{abstract}

\section{Aim}

To identify and quantify the features of bladder cancer in primary care.

\section{Design and setting}

Case-control study, using electronic medical records from UK primary care.

\section{Method}

Participants were 4915 patients aged $\geq 40$ years, diagnosed with bladder cancer January 2000 to December 2009, and 21718 age, sex, and practice-matched controls, were selected from the General Practice Research Database, UK. All clinical features independently associated with bladder cancer using conditional logistic regression were identified, and their positive predictive values for bladder cancer, singly and in combination, were estimated.

\section{Results}

Cases consulted their GP more frequently than controls before diagnosis: median 15 consultations (interquartile range 9-22) versus 8 (4-15): $P<0.001$. Seven features were independently associated with bladder cancer: visible haematuria, odds ratio 34 (95\% confidence interval $[\mathrm{Cl}]=29$ to 41 ), dysuria 4.1 $(95 \% \mathrm{Cl}=3.4$ to 5.0$)$, urinary tract infection 2.2 $(95 \% \mathrm{Cl}=2.0$ to 2.5$)$, raised white blood cell count $2.1(95 \% \mathrm{Cl}=1.6$ to 2.8$)$, abdominal pain $2.0(95 \% \mathrm{Cl}=1.6$ to 2.4$)$, constipation $1.5(95 \%$ $\mathrm{Cl}=1.2$ to 1.9$)$, raised inflammatory markers $1.5(95 \% \mathrm{Cl}=1.2$ to 1.9$)$, and raised creatinine $1.3(95 \% \mathrm{Cl}=1.2$ to 1.4$)$. The positive predictive value for visible haematuria in patients aged $\geq 60$ years was PPV of $3.9 \%(95 \% \mathrm{Cl}=3.4$ to 4.6$)$.

\section{Conclusion}

Visible haematuria is the commonest and most powerful predictor of bladder cancer in primary care, and warrants investigation. Most other previously reported features of bladder cancer were associated with the disease, but with low predictive values. There is a need for improved diagnostic methods, for those patients whose bladder cancer presents without visible haematuria.

\section{Keywords}

bladder cancer; diagnosis; haematuria; primary care.

\section{INTRODUCTION}

Bladder cancer is common worldwide. Worldwide, there are nearly 400000 new cases diagnosed annually, leading to over 150000 deaths. ${ }^{1}$ It is strongly associated with cigarette smoking, and is approximately 2.5 times more common in males. ${ }^{2}$ The incidence rises with age, with the average age at diagnosis being 71 years. ${ }^{2}$ At the time of diagnosis in the UK, approximately $75-85 \%$ patients have a non-invasive tumour, with a recurrence rate of $31-78 \%$, yet with high 5 -year survival of $80-90 \%$. Around $30 \%$ are multifocal. In contrast, once the tumour has invaded muscle, survival is below $50 \% .^{2}$ Emergency presentations occur predominately with advanced disease and have a higher mortality. ${ }^{3}$

More timely diagnosis of bladder cancer may improve outcomes, either by a favourable stage shift or by avoiding emergency presentations. There is some evidence to suggest that better survival is seen in those with shorter times to diagnosis. ${ }^{4.5}$ This may be particularly relevant to the UK and other countries with a gatekeeper system, where access to specialist care requires referral from primary care clinicians. These countries have worse cancer outcomes. ${ }^{6}$ There were an estimated 752 additional bladder cancer deaths in the UK for 1995-1999 when

EA Shephard, PhD, associate research fellow; WT Hamilton, MD, FRCP, FRCGP, professor of primary care diagnostics, Peninsula College of Medicine and Dentistry, Exeter. S Stapley, MSc, BEd, PhD student, School of Social and Community Medicine, University of Bristol, Bristol. RD Neal, PhD, FRCGP, clinical senior lecturer, North Wales Centre for Primary Care Research, Bangor University, Wrexham. P Rose MD, FRCGP, university lecturer, Department of Primary Care Health Sciences, University of Oxford, Oxford. FM Walter, MA, MD, FRCGP, clinical lecturer, Department of Public Health and Primary Care, University of Cambridge, Cambridge. compared with the European average, although some improvement has been seen in selected cancers recently. ${ }^{8}$

There is no accepted screening test for bladder cancer, so diagnosis currently requires presentation with symptoms. This is usually to primary care. 9 However, most diagnostic studies have originated in secondary care. As this population has already been selected for investigation, such results cannot illuminate the selection process. As well as selection and possible recall biases, studies of the referred population inevitably examine patients later in the progress of their disease. The few primary care studies have only examined haematuria. In the first, all 363 patients over 18 with haematuria (either visible or nonvisible) presenting to primary care in Hull, UK, were studied. ${ }^{10}$ Cancers were found in 36 , with $28(7.7 \%$ of the cohort) being bladder tumours. Only three of the 186 with non-visible haematuria transpired to have urological cancer $(1.6 \%)$, in contrast with 32 of the 172 (19\%) with visible haematuria (the minor numerical inconsistency being the authors'). Cancer was more common in patients with a history of urinary tract infection, and slightly so in those with obstructive urinary symptoms. A second study in Belgian primary care identified all patients with visible haematuria, and calculated a positive predictive value (PPV)

\section{Address for correspondence}

William T Hamilton, Peninsula College of Medicine and Dentistry, Veysey Building, Salmon Pool Lane, Exeter, EX2 4SG.

E-mail: willie.hamiltondpcmd.ac.uk

Submitted: 21 December 2011; Editor's response: 15 February 2012; final acceptance: 20 March 2012

\section{British Journal of General Practice}

This is the full-length article (published online 28 Aug 2011) of an abridged version published in print. Cite this article as: Br J Gen Pract 2012; DOI: 10.3399/bjgp12X654560 


\section{How this fits in}

The UK has no screening for bladder cancer so diagnosis in primary care relies on symptomatic presentation. Recognising the early symptoms of bladder cancer could improve the UK's poor mortality outcomes. Existing research has identified haematuria as a significant indicator of risk. No primary care study has looked at identifying multiple features of bladder cancer, including investigations. This study aimed to identify and quantify all significant features in primary care patients. The sample size of nearly 5000 cases identified eight features that were significantly associated with bladder cancer. Results were quantified using a risk assessment tool (RAT) designed to aid GP's referral decision making. Visible haematuria presented the highest risk as a single feature with a positive predictive value (PPV) of 3.9\%, therefore warranting referral. Risks increased with multiple presentation and when combined with abnormal investigations. These results are being fed back in to the re-write of the NICE guidelines.

for bladder cancer of $8.3 \% .^{11}$ Additional symptoms did not alter this figure. In a third study, PPVs for bladder cancer of $8.0 \%$ for males and $3.7 \%$ in females were calculated for visible haematuria in a large cohort aged $16-100$ years. ${ }^{12}$ Several other features of bladder cancer have been described in the secondary care literature, including lower urinary tract symptoms, abdominal pain, and masses. ${ }^{2,13}$

The aim of this study was to identify the symptoms of bladder cancer in primary care, and to quantify them, both singly and in pairs. This should allow improved selection of patients for investigation, reducing the human and economic cost of this disease.

\section{METHOD}

This was a matched case-control study using patients in the UK's General Practice Research Database (GPRD). The GPRD contains approximately 5 million primary care patient records from general practices around the UK, for good geographical representativeness. All primary care consultations are recorded including symptoms, diagnoses, and prescriptions. Investigation results are also documented. Only general practices adhering to stringent data quality recording levels are included in the GPRD.

\section{Cases and controls}

Cases had a first record of bladder cancer lusing one of 20 medical codes for bladder cancer used in the GPRD - available from authors) between January 2000 and December 2009 inclusive. They were aged $\geq 40$ years, and had a minimum of 1 year of data before diagnosis. The first instance of a bladder cancer code was assigned the date of diagnosis: the index date. The index date for controls was the index date for their matched case. Up to five controls were matched on sex, general practice, and to 1 year of age of the case. The GPRD only provides the year of birth (to protect anonymisation), so an arbitrary birth date of 1 July was assigned to everyone. Exclusion criteria were: metastatic cancer of the bladder from a non-bladder primary, diagnosis before 2000 , or no consultations in the year before diagnosis.

\section{Selection of investigations and symptom variables}

A list of symptoms, signs, and investigations (called 'features' from now on) potentially associated with bladder cancer was compiled from the literature search, augmented by viewing material from bladder cancer support organisations and online chat rooms. Internet search terms included 'bladder cancer', 'bladder symptoms', and 'early signs/indications'. Visible and non-visible haematuria were studied separately. Only codes specifying the word 'microscopic' were assigned to the latter group, so generic codes such as the single word 'haematuria' were assumed to be visible haematuria. For each feature a list of relevant medical codes was assembled from the GPRD's master list of over 100000 codes. Occurrences of these were identified in the year before the index date. Repeated consultations for the same complaint were also identified. Also identified were all codes for fractures as a test for any recording bias between cases and controls (making the assumption that the fracture rate would be approximately equal). Variables were retained only if they occurred in at least $5 \%$ of either cases or controls (this was always cases). Investigation results were deemed to be abnormal if they fell outside their local laboratory's normal range for analysis. Patients with a normal laboratory result were grouped with those who had not been tested.

\section{Analysis and statistical methods}

This followed the methods used in several previous studies. ${ }^{14}$ The main analytical method was conditional logistic regression. Analysis was performed in three stages. All variables associated with cancer with a 
$P$-value of $\leq 0.1$ in univariable analysis were retained. In the second stage, variables were grouped for multivariable analysis, collecting together variables that were similar, such as visible and non-visible haematuria, using a $P$-value threshold of $\leq 0.05$. The final stage of multivariable analysis used all variables surviving the previous stages, and used a $P$-value threshold of 0.01 . All excluded variables were checked against the final model.

Positive predictive values (PPVs) were estimated for features shown to be independently associated with cancer in the multivariable analysis. This was repeated for pairs of symptoms and for second attendances with the same symptom. PPVs were estimated using Bayes' theorem, whereby the prior odds $x$ likelihood ratio $(L R)=$ posterior odds. ${ }^{15}$ The prior odds used were the age-specific national incidence of bladder cancer for 2008, expressed as odds. To enable a calculation of PPVs for the consulting population, the proportion of the control population who had not consulted in the year before diagnosis was estimated. Of 23804 eligible controls, 2086 (8.8\%) had not consulted; so PPVs were divided by 0.912 to give the figure for the consulting population (Figure 1).

As the number of cases in the GPRD

Figure 1. Application of exclusion criteria.

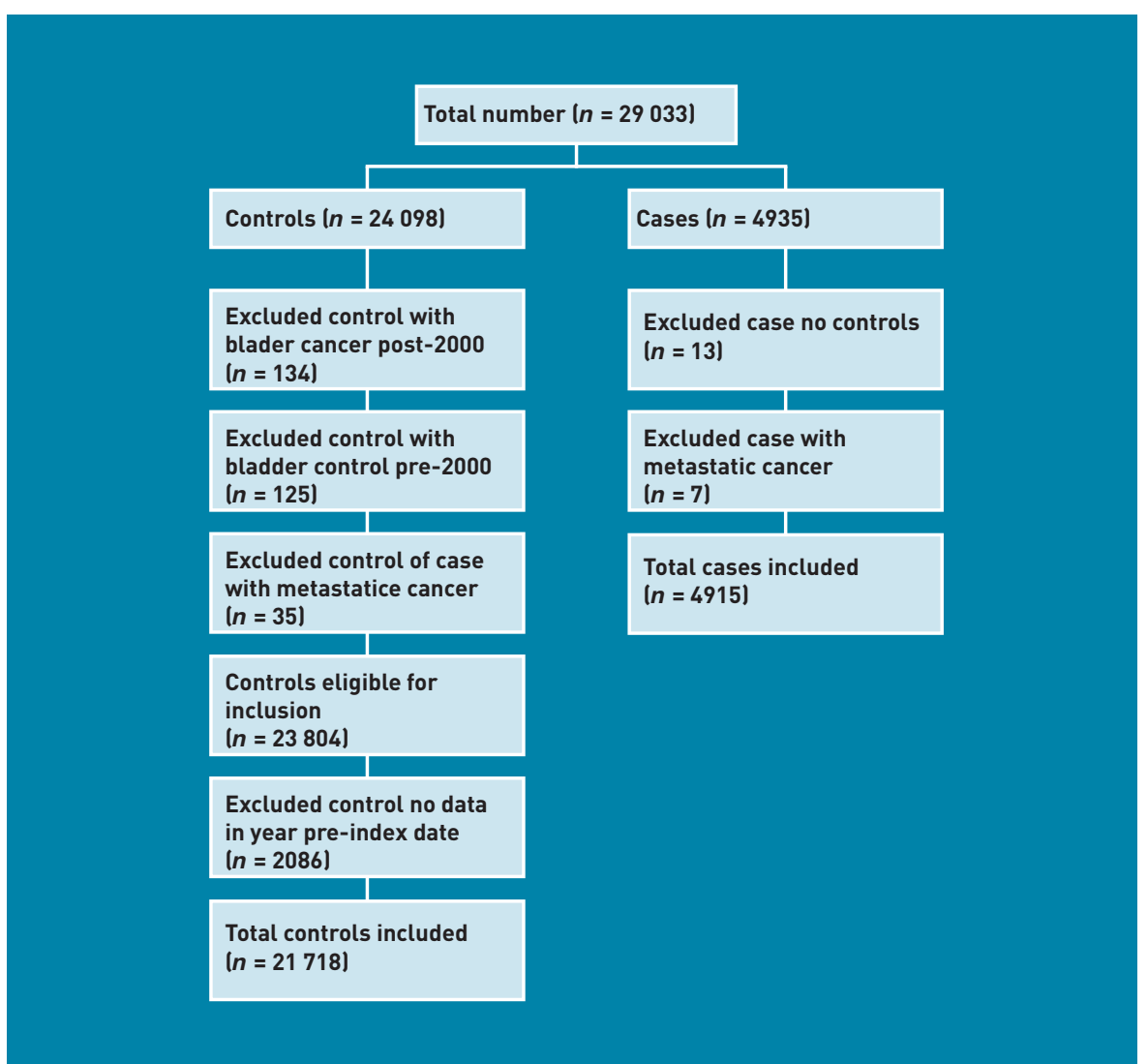

were fixed, a power calculation was performed rather than a sample size calculation. Thus, 5000 cases and 20000 controls (the estimates initially provided by the GPRD) provided $>99.9 \%$ power $(5 \%$ two-sided alphal to detect a change in a rare variable from $1 \%$ in cases and $2 \%$ of controls. For a commoner variable, the study had $>89 \%$ power to detect a change in prevalence of $20 \%$ in cases to $18 \%$ in controls. Data analysis was conducted using Stata software (version 11).

\section{RESULTS}

The GPRD provided 29033 patients (4935 cases; 24098 controls). Application of the exclusion criteria is shown in Figure 1. leading to a final number of 2663314915 cases; 21718 controls).

Patient demographic and consultation information is given in Table 1. Cases consulted significantly more frequently than controls in the year before diagnosis ( $P \geq 0.001$; rank sum test).

\section{Clinical features}

Forty-three symptoms and 104 abnormal test results were considered initially. Six symptom and seven abnormal test variables were present in at least $5 \%$ of cases. Only $2.6 \%$ of cases in the study had a record of non-visible haematuria. Features associated with bladder cancer in univariable analysis are shown in Table 2. Raised inflammatory markers were defined as being above the local laboratory's quoted normal range for erythrocyte sedimentation rate or C-reactive protein. Table 2 also shows the multivariable analysis. All variables retained an independent association with cancer, with a $P$-value $<0.001$ in the final multivariable model. No interaction terms were found.

From a total of 4915 cases, 3621 (74\%) had at least one of the features from the final model in Table 2 recorded in their notes. The proportion of patients with a recorded fracture did not differ between cases $(n=71,1.45 \%)$ and controls $(n=316$, $1.46 \%)$

\section{Positive predictive values}

Figure 2 shows the PPVs for individual, combined, and repeat features, for patients aged $\geq 60$ years.

The LRs were largely similar between the two age groups ( $40-59$ years and $\geq 60$ years) except for visible haematuria, which had a stronger association with cancer in younger patients (LR 420) compared with older patients (LR 53). The incidence of bladder cancer is approximately tenfold 
Table 1. Patient demographics and consultation rates in the year before diagnosis

\begin{tabular}{|c|c|c|c|c|c|c|}
\hline & \multicolumn{3}{|c|}{ Case } & \multicolumn{3}{|c|}{ Control } \\
\hline & $\begin{array}{c}\text { Male } \\
n=3563 \text { l }\end{array}$ & $\begin{array}{l}\text { Female } \\
\text { (n=1352) }\end{array}$ & $\begin{array}{c}\text { Total } \\
\text { ( } n=4915)\end{array}$ & $\begin{array}{c}\text { Male } \\
(n=15 \text { 452) }\end{array}$ & $\begin{array}{l}\text { Female } \\
(n=6266)\end{array}$ & $\begin{array}{c}\text { Total } \\
\text { ( } n=21718)\end{array}$ \\
\hline $\begin{array}{l}\text { Median age at } \\
\text { diagnosis, years (IQR) }\end{array}$ & 73 (65-80) & 75 (67-82) & $74(66-80)$ & $73(66-79)$ & 75 (67-82) & 74 (66-80) \\
\hline $\begin{array}{l}\text { Median number of } \\
\text { consultations (IQR) }\end{array}$ & 14 (9-22) & 15 (10-23) & 15 (9-22) & $8(4-15)$ & $9(4-15)$ & 8 (4-15) \\
\hline
\end{tabular}

lower in patients aged 40-59 than in those aged $\geq 60$ years, so PPVs in the younger age group are also approximately tenfold lower for symptoms other than visible haematuria (data not shown). The PPV of visible haematuria in the 40-59 year age group was 3.1\% $195 \%$ confidence interval $[\mathrm{Cl}]=1.0$ to 9.8$)$

\section{DISCUSSION}

This is the first study from primary care to identify all the clinical features of patients with bladder cancer in that setting. It was possible to quantify these features using the most useful metric for clinicians: the PPV for a consulting patient. Four symptoms were identified that were both common and significantly associated with bladder cancer, from an original list gathered from several sources as well as conventional publications. Also confirmed was an association for urinary tract infection and three standard investigations. For a patient aged $\geq 60$ years describing haematuria to his or her GP, the risk of cancer was $3.9 \%$. Risks increased with multiple and repeated symptoms. In the absence of visible haematuria or dysuria, however, risks were consistently low.

\section{Table 2. Clinical features of bladder cancer (all ages)}

\begin{tabular}{|c|c|c|c|c|}
\hline Feature & $\begin{array}{c}\text { Cases, } n(\%) \\
n=4915\end{array}$ & $\begin{array}{c}\text { Controls, } n(\%) \\
n=21718\end{array}$ & $\begin{array}{c}\mathrm{LR}^{\mathrm{a}} \\
(95 \% \mathrm{CI})\end{array}$ & $\begin{array}{c}\text { ORb } \\
(95 \% \mathrm{Cl})\end{array}$ \\
\hline \multicolumn{5}{|l|}{ Symptoms } \\
\hline Visible haematuria & 2595 (53) & $196(1)$ & 59 (51 to 67 ) & $34(29$ to 41$)$ \\
\hline Dysuria & 444 (9) & $209(1)$ & $9.4(8.0$ to 11$)$ & 4.1 (3.4 to 5.0 ) \\
\hline Abdominal pain & 358 (7) & $787(4)$ & 2.0 (1.8 to 2.3$)$ & $2.0(1.6$ to 2.4$)$ \\
\hline Constipation & $286(6)$ & 708 (3) & $1.8(1.6$ to 2.0$)$ & $1.5(1.2$ to 1.9$)$ \\
\hline \multicolumn{5}{|l|}{ Disease } \\
\hline Urinary tract infection & 835 (17) & 705 (3) & $5.2(4.8$ to 5.8$)$ & 2.2 (2.0 to 2.5 ) \\
\hline \multicolumn{5}{|l|}{ Investigations } \\
\hline Raised creatinine & 660 (13) & $1668(8)$ & $1.8(1.6$ to 1.9$)$ & $1.3(1.2$ to 1.4$)$ \\
\hline Raised inflammatory markers & $293(6)$ & 717 (3) & $1.8(1.6$ to 2.1$)$ & $1.5(1.2$ to 1.9$)$ \\
\hline Raised white blood cell count & $250(5)$ & 401 (2) & 2.8 (2.4 to 3.2$)$ & 2.1 (1.6 to 2.8$)$ \\
\hline
\end{tabular}

\section{Strengths and limitations}

The main strength of this study is its primary care setting. This is where the clinical problem exists: the decision of whom to select for investigation for possible bladder cancer. A further strength is that the symptom and investigation variables were collected before the diagnosis was established. Such a design eliminates recall bias (which can affect studies reporting retrospectively collected data), although it may add recording bias (see below). The study was large and generalisable, as GPRD data are representative of patients across the UK.

The main limitation of using electronic records as the data source is reliance upon the quality of the doctors' recording. Patient records have been widely used in similar cancer studies before, including GPRD data. ${ }^{12,14,16-19}$ Symptoms may be unvoiced or unrecorded, but this only matters if one of these is more common in either of the two groups, cases or controls. There was reason to suspect this, and the test - the frequency of recording of fractures in cases and controls - supported this. If underrecording is equally prevalent between cases and controls, the likelihood ratios and PPVs will still be correct, although the $74 \%$ of patients with at least one feature of bladder cancer will be an underestimate. Indeed, PPVs from electronic primary care studies like this one largely match those derived from the few truly prospective studies. 20,21 The matched design meant that controls were from the same practice as cases, which should reduce recording bias. Matching makes it impossible to study the matched variables directly, although the study was large enough for sub-analyses by sex and age-group to be performed, sidestepping that potential weakness. Furthermore, laboratory data have been transmitted automatically to practices since around 2000, so there should be no recording bias present for these variables, which is why that date was chosen for the start of the study.

Only symptoms previously reported with bladder cancer were studied, although including patient groups and online chat rooms in a literature search made it unlikely that any salient symptoms were omitted. The investigations were even more inclusive: all primary care tests entered the analysis, although few were found to be significant. Verification bias occurs when testing is more commonly performed in one group (generally cases) than the other, so positive results are more common as a result of more testing las well as any true 
Figure 2. Positive predictive values for bladder cancer in patients aged $\geq 60$ years, for individual, paired, and repeated features. association). This could have been possible for non-visible haematuria, whereby a doctor may be more likely to perform urinalysis in a patient with bladder cancer. This variable was only present is $2.6 \%$ of cases, so was omitted from the main analysis.

Finally, the methods for calculating PPVs are innovative, although are now wellestablished. ${ }^{14}$ It is impossible to estimate PPVs using only the information within a case-control study las the proportion of cases - the prior odds - is higher than in the population as a whole). In cancer studies, excellent national incidence statistics are available as a proxy for the prior odds. Indeed, these national figures may actually be superior to the prior odds

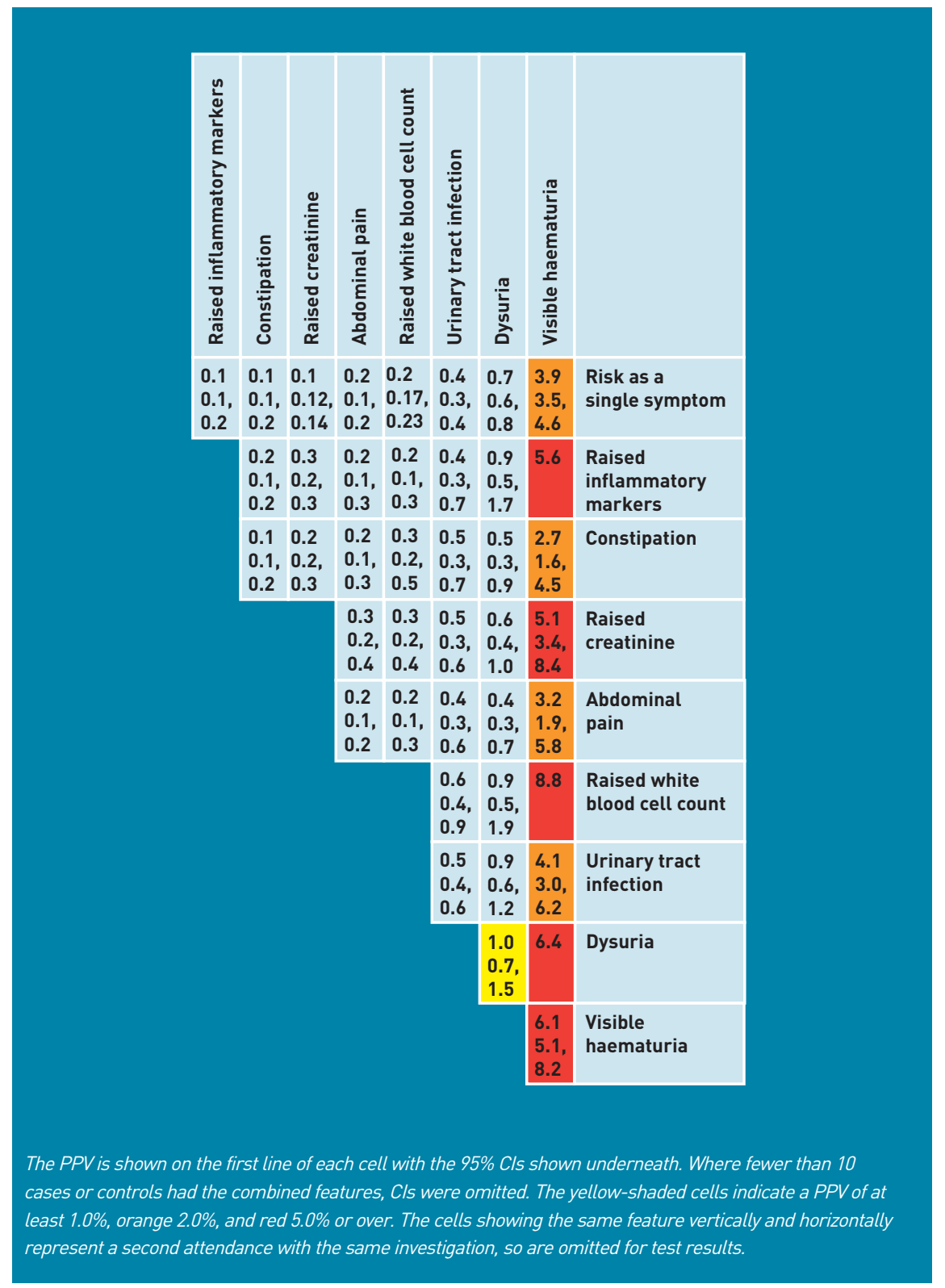

derived from any cohort study in primary care, as the number of cancers in such a study would perforce be very small.

\section{Comparison with previous literature}

The only symptom of bladder cancer previously reported from primary care is visible haematuria. A study of patients in a UK open-access clinic for both visible and non-visible haematuria identified several additional factors increasing the likelihood that a urological cancer was present. ${ }^{10}$ These included age and sex, several lower urinary tract symptoms, and a history of previous urinary tract infection. No PPVs were estimated. The Belgian study estimated a PPV of $10.3 \%$ for urological cancer with visible haematuria presented to primary care; $69 \%$ of these were bladder cancers, giving an approximate 7\% PPV for bladder cancer. The figure of $3.1 \%$ for ages 40-59 years was based on very few controls reporting visible haematuria to their doctors and so has very wide Cls. However, those aged $\geq 60$ years had a PPV of $3.9 \%(95 \% \mathrm{Cl}=3.4$ to 4.6$)$ as numbers were much higher. Overall, the Belgian study had considerably fewer cases only 87 - and thus had wide Cls for all their risk estimates. Their cases of cancer were identified from a cancer registry quite separate from the collection method for those reporting haematuria. Further, Belgian patients may access urologists directly, so those patients reporting haematuria to primary care may be a selfselected group. ${ }^{22}$ Few clinicians, or their patients, would disagree with investigation for cancer at a risk of $3.9 \%$, and many would choose investigation at a lower value. Therefore, this study supports investigation for possible bladder cancer in all the over40 s with visible haematuria. This is the view of NICE guidance and a recent review, but without primary care data to substantiate them. ${ }^{23,24}$ A 2003 systematic review of the economics of bladder cancer identified six papers examining the cost-effectiveness of investigation for bladder cancer. ${ }^{25}$ In a population with a prevalence of $1 \%$ there was an estimated cost of $\$ 170000$ per case identified; this figure fell to $\$ 12000$ per case if the prevalence were $15 \% .^{26}$ No recent evaluation has been reported, although it is likely the 'threshold' level for investigation should be above 1\%, on health-economic grounds at least.

A link between urinary tract infection and bladder cancer has been reported before: either with infection predisposing to cancer, or infection being a presenting feature of a cancer. 27 This study only addressed the 
latter aspect, showing a moderate increase in the risk of cancer when urinary infection was recorded by a GP. Also found were independent associations for dysuria as a symptom, and for raised inflammatory markers or leukocytosis, all of which may be present with urinary infection. Indeed, this distinction between urine infection and dysuria may reflect the data source, in that different doctors may record similar clinical scenarios differently. Nonetheless, it is clear that such symptoms are relevant in diagnosis of possible bladder cancer. A classic cause of delay in bladder cancer is when a patient has repeated urine infections, each successfully treated, but without bladder cancer being considered. The study results show that urological anecdote has some basis in truth. Two other symptoms were roughly twice as common in cases as controls: constipation and abdominal pain. However, as isolated symptoms - or even together - their predictive values were very small, so it is unlikely they will provide much help in selection of patients for investigation.
Decreased renal function has previously been reported with bladder cancer. ${ }^{28}$ It cannot be determined if the patients had obstructive nephropathy, to account for the higher percentage of cases in this study with raised creatinine.

\section{Implications for research and practice}

The findings support investigation of all patients aged $>40$ years with visible haematuria. It is highly likely that such a recommendation would be supported on economic as well as clinical grounds. The only other clinical scenario with a risk of cancer above $1 \%$ was repeated attendances with dysuria in patients $>60$ years. Figure 2 adds to the other cancer 'Risk Assessment Tools' (RATs) that are being introduced into UK primary care. ${ }^{29}$ Currently, only colorectal and lung RATs are being disseminated. If they are found to be helpful, bladder could easily be added. This may best be done allied with prostate, already published in the $B J G P^{30}$ and kidney, which is currently being studied, into a single urological RAT.

\section{Funding}

This study was funded by the NIHR Programme Grants for Applied Research funding scheme, RP-PG-0608-10045. William $T$ Hamilton was part-funded by a NIHR post-doctoral fellowship.

\section{Ethical approval}

Independent Scientific Advisory Committee: protocol 09-110.

\section{Provenance}

Freely submitted; externally peer reviewed.

\section{Competing interests}

The authors have declared no competing interests.

\section{Acknowledgements}

The authors would like to acknowledge the contribution to the research presented in this paper made by the Discovery Programme Steering Committee comprising: Roger Jones (chair); Jonathan Banks; Alison Clutterbuck; Jon Emery; Joanne Hartland; Sandra Hollinghurst; Maire Justice; Jenny Knowles; Helen Morris; Tim Peters; Greg Rubin.

\section{Discuss this article}

Contribute and read comments about this article on the Discussion Forum: http://www.rcgp.org.uk/bjgp-discuss 


\section{REFERENCES}

1. Jemal A, Bray F, Center MM, et al. Global cancer statistics. CA Cancer J Clin 2011; 61(2): 69-90.

2. Mowatt G, Zhu S, Kilonzo M, et al. Systematic review of the clinical effectiveness and cost-effectiveness of photodynamic diagnosis and urine biomarkers (FISH, ImmunoCyt, NMP22) and cytology for the detection and follow-up of bladder cancer. Health Technol Assess 2010; 14(4): 1-131.

3. National Audit Office. Delivering the cancer reform strategy. London: The Stationery Office, 2010.

4. Wallace D, Bryan R, Dunn J, et al. Delay and survival in bladder cancer. BJU Int 2002; 89(9): 868-878

5. Liedberg F, Anderson H, Mansson A, Mansson W. Diagnostic delay and prognosis in invasive bladder cancer. Scand J Urol Nephrol 2003; 37(5): 396-400.

6. Vedsted $\mathrm{P}$, Olesen $\mathrm{F}$. Are the serious problems in cancer survival partly rooted in gatekeeper principles? Br J Gen Pract 2011; 61(589): 512-513.

7. Abdel-Rahman M, Stockton D, Rachet B, et al. What if cancer survival in Britain were the same as in Europe: how many deaths are avoidable? Br J Cancer 2009; 101(S2): S115-124

8. Coleman MP, Forman D, Bryant H, et al. Cancer survival in Australia, Canada, Denmark, Norway, Sweden, and the UK, 1995-2007 Ithe International Cancer Benchmarking Partnership): an analysis of population-based cancer registry data. Lancet 2011; 377(9760): 127-138.

9. Hamilton W. Cancer diagnosis in primary care. Br J Gen Pract 2010; 60(571): $121-128$.

10. Summerton N, Mann S, Rigby A, et al. Patients with new onset haematuria: assessing the discriminant value of clinical information in relation to urological malignancies. Br J Gen Pract 2002; 52(477): 284-289.

11. Bruyninckx R, Buntinx F, Aertgeerts B, Van Casteren V. The diagnostic value of macroscopic haematuria for the diagnosis of urological cancer in general practice. Br J Gen Pract 2003; 53(486): 31-35.

12. Jones R, Latinovic R, Charlton J, Gulliford MC. Alarm symptoms in early diagnosis of cancer in primary care: cohort study using General Practice Research Database. BMJ 2007; 334(7602): bmj.39171.637106.AE.

13. Khadra MH, Pickard RS, Charlton M, et al. A prospective analysis of 1,930 patients with hematuria to evaluate current diagnostic practice. J Urol 2000; 163(2): 524-527.

14. Hamilton W. The CAPER studies: five case-control studies aimed at identifying and quantifying the risk of cancer in symptomatic primary care patients. $\mathrm{Br}$ Cancer 2009; 101(S2): S80-86.

15. Knottnerus JA. The evidence base of clinical diagnosis. London: BMJ Books, 2002
16. Hamilton W, Kernick D. Clinical features of primary brain tumours: a casecontrol study using electronic primary care records. Br J Gen Pract 2007, 57(542): 695-699.

17. Hamilton W, Lancashire R, Sharp D, et al. The importance of anaemia in diagnosing colorectal cancer: a case-control study using electronic primary care records. Br J Cancer 2008; 98(2): 323-327.

18. Lawrenson R, Logie J, Marks C. Risk of colorectal cancer in general practice patients presenting with rectal bleeding, change in bowel habit or anaemia. Eur J Cancer Care 2006; 15(3): 267-271.

19. Tate AR, Martin AGR, Murray-Thomas T, et al. Determining the date of diagnosis - is it a simple matter? The impact of different approaches to dating diagnosis on estimates of delayed care for ovarian cancer in UK primary care. BMC Medical Research Methodology 2009; 9: 42. doi:10.1186/1471-2288-9-42.

20. Du Toit J, Hamilton W, Barraclough K. Risk in primary care of colorectal cancer from new onset rectal bleeding: 10 year prospective study. BMJ 2006; 333(7558) $69-70$.

21. Friedman GD, Skilling JS, Udaltsova NV, Smith LH. Early symptoms of ovarian cancer: a case-control study without recall bias. Fam Pract 2005; 22(5): 548-553.

22. Hay A, Hamilton W, Sharp D, et al. Macroscopic haematuria and urological cancer. Br J Gen Pract 2003; 53(488): 241-242.

23. National Institute for Health and Clinical Excellence. Referral guidelines for suspected cancer. London: NICE, 2005.

24. Kelly JD, Fawcett DP, Goldberg LC. Assessment and management of non-visible haematuria in primary care. BMJ 2009; 338: a3021.

25. Botteman MF, Pashos CL, Redaelli A, et al. The health economics of bladder cancer: a comprehensive review of the published literature. Pharmacoeconomics 2003; 21(18): 1315-1330.

26. Corwin HL, Silverstein MD. The diagnosis of neoplasia in patients with asymptomatic microscopic hematuria: a decision analysis. J Urol 1988; 139(5): 1002-1006

27. Mommsen S, Aagaard J, Sell A. Presenting symptoms, treatment delay and survival in bladder cancer. Scand J Urol Nephrol 1983; 17(2): 163-167.

28. El-Tabey NA, Osman Y, Mosbah A, et al. Bladder cancer with obstructive uremia: oncologic outcome after definitive surgical management. Urology 2005; 66(3): $531-535$.

29. Department of Health. Improving outcomes: a strategy for cancer. London: DoH, 2011

30. Hamilton W, Sharp D, Peters TJ, Round A. Clinical features of prostate cancer before diagnosis: a population-based, case-control study. Br J Gen Pract 2006; 56(531): 756-762. 